\title{
Laser-Assisted Uvulopalatoplasty (LAUP)
}

\author{
Sv. Vasileva', PI. Nedev², S. Stoyanov' \\ M. Milkov², R. Benchev ${ }^{3}$ \\ 'Department of Otorhinolaryngology, \\ Medical Institute of the Ministry of Interior, Sofia \\ ${ }^{2}$ Department of Otorhinolaryngology, \\ Medical University „Prof. Dr. Paraskev Stoyanov“, Varna \\ "Hill Clinic" Ltd, Sofia
}

\section{Abstract:}

Surgical treatment options for patients suffering from Sleep Disordered Breathing marked a significant development in recent decades. To date, there is a huge variety of methods and techniques, developed to address this problem. The Introduction of laser and radiofrequency surgery in the daily practice, make possible the so called "minimally invasive surgery". It can be done under local anaesthesia and in an outpatient setting.

The purpose of this article is to introduce one of the most modern methods - Laser-Assisted Uvulopalatoplasty (LAUP). This is a relatively new method and the experience with it in Bulgaria is limited - for a lot of specialists the method is largely unknown as technical implementation and opportunities. Our purpose is to present this useful, relatively easy to perform and highly efficient method and we hope that more Bulgarian ENT specialists will begin to use it in their everyday practice.
\end{abstract}

\section{Introduction}

Laser -Assisted Uvulopalatoplasty (LAUP) is presented for the first time by Dr Yves-Victor Kamami in 1986, but in 1993 it began to be widely applied in practice. In 1997, the American Academy of Otolaryngology Head and Neck Surgery includes LAUP as a procedure for the treatment of OSA ${ }^{\mathrm{a}}$.

This type of surgery, as an independent procedure, is indicated in patients with the so-called "Flat type velopharynx", i.e. when the obstruction of the upper airways, comes with priority from the soft palate - the uvula and / or arcs and there is no obstruction by the tongue (FTP I - II). When there is tongue obstruction (FTP III - IV), hypertrophy of the tonsils (FTS III - IV) or obstruction by the lateral columns, LAUP may be used as an element of multilevel surgery ${ }^{\mathrm{b}}$.

\section{Surgical technique}

From the literature we know two basic methods to perform this type of surgery. The first, known as the French method (presented by Kamami in 1990') is suitable for patients in whom this is the first intervention on the soft palate. 


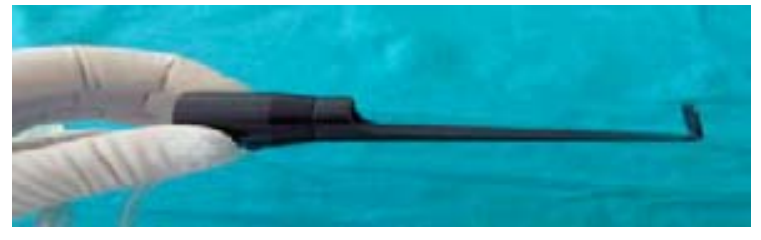

Fig. 1. Hand-piece for the soft palate and uvula (with "backstop" for the protection of the lining on the back wall of the oropharynx)

We work with CO2 LASER "DEKA" (Fig. 1 and 2) most commonly by the method of Kamami.

\section{Surgical steps}

1. Local anaesthesia: Surgery can be performed under local anaesthesia. 5-10 minutes before the intervention are made 2 separate applications of Lidocaine spray. Immediately prior to vaporization, even when working under general anesthesia, we infiltrate Lidocaine and Adrenaline $(1: 100000)$ in 6 points - two above the uvula in the midline of the soft palate and in 2 points laterally over the anterior arcs in order to reduce bleeding.

2. The work begins in continuous mode $15 \mathrm{~W}$. (Fig. 3). In massive palates, the power can be increased manually to $20-25 \mathrm{~W}$.

3. Evaporation of the domed fragments of the lining of the rear palatal arcs to the level of the muscles and of up to $1 \mathrm{~cm}$ laterally from the uvula. Parts of tonsillar arches and tonsils can also be treated (Fig. 4).

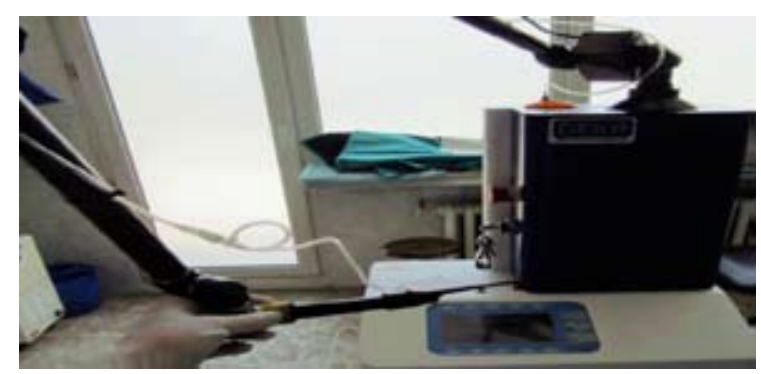

Fig. 2. CO2 LASER "DEKA"

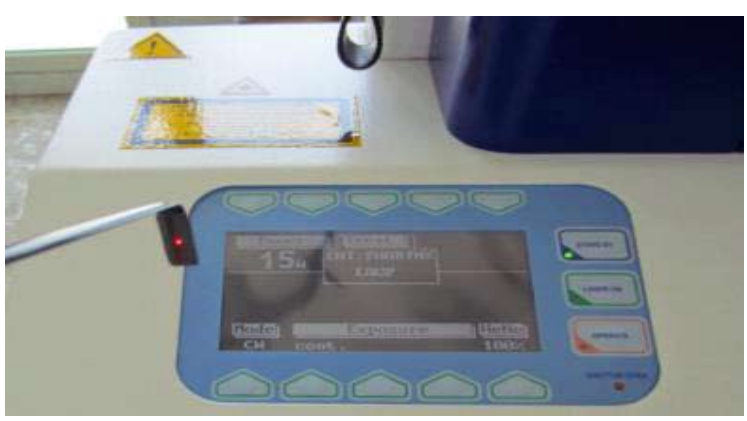

Fig. 3. Display of the CO2 LASER Parameters for LAUP
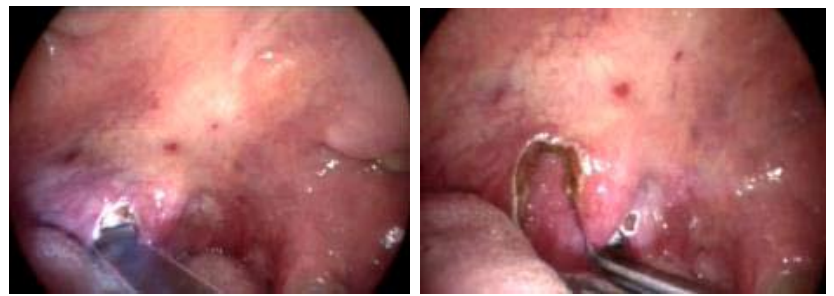

Fig. 4. Evaporation of the rear palatal arches

4. Excision of the distal parts $(2 / 3)$ of the uvula gradually on both sides (Figure 5).
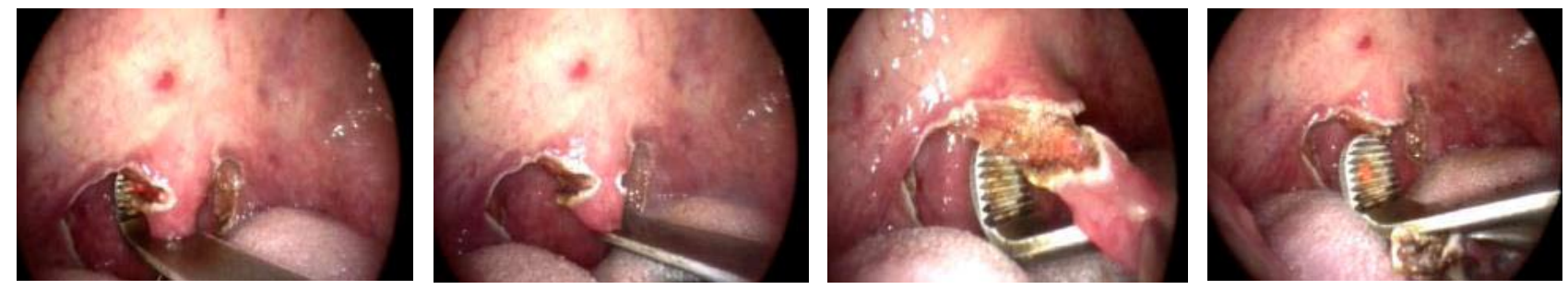

Fig. 5. Excision of the uvula

Fig. 6. British method - (The figure is from Friedman M.: Sleep apnea and snoring: surgical and non-surgical therapy 2009 )

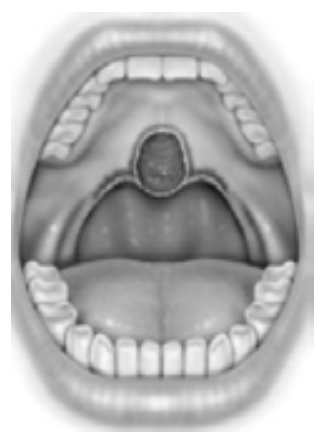


Bleeding usually is insignificant and can be controlled by changes in the laser beam focus. Extremely rare is hemorrhage that can require the use of additional methods of haemostasis. Healing of the wound surface occurs usually between the second and third week (14 and 21 days). Patients have pain (not very strong) until a week after surgery. If necessary, the procedure can be repeated.

In 1994 Woolford and Farrington ${ }^{\text {d present the }}$ modification of this method, known as the British technique (Fig. 6). Most often it is used as a second intervention after LAUP by the method of Kamami.

\section{Surgical technique}

The scanning beam of the laser is used with a power setting of $20 \mathrm{~W}$. Approximately $2 \mathrm{~cm}$ of mucosa and submucosa on the oral side of the soft palate and uvular base is vaporized. The underlying median raphe and palatal musculature should be carefully preserved. The surface is allowed to contract and form scar secondary, which leads to further hardening of the palate.

An advantage of LAUP is also the fact that in addition to the removal of mucosal parts, by laser is caused a special form of a surface inflammation, which leads to the formation of centripetal fibrosis and thickening of the tissues, resulting in a further decrease in their ability to vibrate. Specific caution should be paid to avoid a circumferential wound or continuous raw surface that may cause pharyngeal contraction and stenosis.

LAUP can also be performed under local anesthesia in an outpatient setting e f but it should be kept in mind that during the early post-operative period is possible to development of edema of the upper respiratory tract ${ }^{\mathrm{g}}$, requiring control and monitoring of oxygen saturation for at least 48 hours. In this regard, more authors recommend LAUP to be performed after hospitalization. The procedure is less painful compared to classical uvulopharyngopalatoplasty (UPPP), but during the first few days most patients need analgesics. Coexisting gastro-esophageal reflux also should be treated. Patients should be monitored and re-evaluated at least 6 weeks after surgery. Based on the literature data for the optimal effect from LAUP patients should undergo between 2 and 4 treatments every 4-6 weeks. Up to now we didn't have to repeat the procedure.

\section{Complications}

LAUP is technique with a high level of safety. The most frequently reported complications are: foreign body sensation, dryness in the pharynx, postoperative bleeding, infection, palatal or velopharyngeal incompetence and very rarely - naso-pharyngeal stenosis. Average rate of complications is about $3.5 \% \mathrm{~h}$. Most common are haemorrhage - 2-3\%, followed by infection - $1 \%$. Velopharyngeal incompetence was observed in less than $1 \%$ of patients and often is transitional. Cases of velopharyngeal stenosis are also, but their frequency is very low.

\section{Effectiveness}

In 1990 Kamami reported $77 \%$ complete or nearcomplete resolution of snoring in non-apnoeic snorers i. A follow-up study by Walker and colleagues echoed these results. In their series, $60 \%$ of 170 snorers had total or near-total elimination of snoring, with an additional $29 \%$ who reported partial improvement ${ }^{i}$. Average reported success rate of the procedure in terms of habitual snoring is between 60 and $84 \%$. A number of authors have reported a similar percentage of the improvement in quality of life and partner's relationships (72\% satisfaction of the partner $)^{\mathrm{j} k}$.

The correct patient selection is an integral element in achieving good outcomes and patient satisfaction. In well selected cases, reported effectiveness, according to the criteria of Sher is $57 \%$ in mild, $48 \%$ in moderate and $46 \%$ in patients with severe degrees of OSA ${ }^{1 \mathrm{~m}}$.

There are also reports a significantly lower success rates (objective, after polysomnography) - 27\%, emphasizing that the positive subjective evaluation of patients greatly exceeds the objective results ${ }^{\mathrm{n}}$.

There are also reports of objective deterioration of patients with OSA after LAUP - Finkelstein and colleagues in 2002 during investigation of 26 patients reported $31 \%$ increase of RDI postoperatively. They suggested that the reason is velopharyngeal stenosis as a result of progressive palatal fibrosis. ${ }^{\circ}$

In the operated by us 24 patients (men), between 6 and 9 months after surgery, we recorded ${ }^{\mathrm{p}}$ :

- $92 \%$ efficiency in terms of snoring (subjective evaluation from history and questionnaires). Only two patients' partners reported that although at lower intensity, snoring persists for more than 3-4 nights during the week. 
- Before operations 6 patients were with ESS over 10 ie increased daytime sleepiness. After the interventions ESS was less than 10 for all included in this study patients - i.e. $100 \%$ efficiency on the basis of subjective evaluation.

- The mentioned in the last paragraph 6 patients before the operation held polysomnography (polygraphy) and all of them were with AHI over 5 (9 to 28). After the operation, reduction of AHI with $50 \%$ or more was achieved in 4 of them (67\% success rate).

Among the operated patients we didn't have cases with postoperative bleeding. The pain was more pronounced than after RF-uvulopalatoplasty (RF UPP), but significantly less than after UPPP. There was need of analgesics during the first 1-2 days. Seven patients had a pronounced swelling of the soft palate until 4-6 days after operations and reported for foreign body sensation in their throat. In four of them the problem passed spontaneously, while in three there was a need of corticosteroids for a period of 3 days. In two patients was found an infection at the wound surface, respectively 5 and 7 days after surgery (at home), but the inflammation was easily cured by topical antiseptic solutions and oral antibiotic.

\section{Conclusion}

Our experience with LAUP is still comparatively limited, but based on our results we can state that LAUP is a relatively easy method with high level of safety, low risk of postoperative complications. It is well tolerated by patients and is highly effective when used for the right indications. Extremely important in this type of surgery is to avoid circumferential wound (scars). Long-term satisfaction after LAUP is in strong correlation with two factors - proper selection of patients and precise performance.

LAUP is a technique with proven place in the treatment of snoring and sleep apnea. The reduction of the effect over time and the possible need to repeat the procedure are the only factors limiting its use in clinical practice.

\section{References}

a Walker RP. Laser assisted uvulopalatoplasty. In: Snoring and Obstructive Sleep Apnea, 3rd edn . 2003: 144-50

b Finkelstein Y, Shapiro-Feinberg M, Stein G et al: Uvulopalatopharyngoplasty vs laser-assisted uvulopalatoplasty. Arch Otolaryngol Head Neck Surg 123: 265-276, 1997

c Kamami Y. Laser $\mathrm{CO}_{2}$ for snoring, preliminary results. Acta Otorhinolaryngol Belg 1990; 44: 45-56

d Woolford T, Farrington T. Laser-assisted uvulopalatoplasty - the British method. Oper Tech Otolaryngol Head Neck Surg 1994; 5: 292-93

e Remacle M, Betsch C, Lawson G et al: A new technique for laser-assisted uvulo(palato)plasty decision-tree analysis and results. Laryngoscope 109: 763-768, 1999

f Mickelson SA, Ahuja A: Short-term objective and long-term subjective results of laser

g Esclamado RM, Glenn MG, McCulloch TM, et al. Perioperative complications and risk factors in the surgical treatment of obstructive sleep apnea syndrome. Laryngoscope 1989; 99: 1125-29

h Walker RP, Gopalsami C. Laser-assisted uvulopalatoplasty: postoperative complications. Laryngoscope 1996; 106: 834-8

i Walker RP, Grigg-Damberger MM, Gopalsami C, et al. Laserassisted uvuloplasty for snoring and obstructive sleep apnea: resultsin 170 patients. Laryngoscope 1995; 105: 938.43

j Armstrong MW, Wallace CL, Marais J. The effect of surgery upon the quality of life in snoring patients and their partners: a between-subjects case-controlled trial . Clin Otolaryngol Allied Sci 1999; 24: 510-22

k Prasad KR, Premraj K, Kent SE, Reddy KT. Surgery for snoring:are partners satisfied in the long run? Clin Otolaryngol Allied Sci 2003; 28: 497-502

1 Mickelson SA, Ahuja A. Short-term objective and long-term subjective results of laser-assisted uvuloplatoplasty for obstructive sleep apnea . Laryngoscope 1999; 109: 362-7

m Walker RP, Garrity T, Gopalsami C. Early polysomnographic findings and long-term subjective results in sleep apnea patients treated with laser-assisted uvulopalatoplasty. Laryngoscope 1999; 109: 1438.41

n Ryan CF, Love LL. Unpredictable results of laser assisted uvulopalatoplasty in the treatment of obstructive sleep apnoea. Thorax 2000; 55: $399-404$

o Finkelstein Y, Stein G, Ophir D, et al . Laser-assisted uvulopalatoplasty for the management of obstructive sleep apnea Arch Otolaryngol Head Neck Surg 2002; 128: 429-34

p Vasileva Sv.: Modern methods for surgical treatment of Sleep Disordered Breathing - Dissertation - 2014; 22-24: 87-88 


\title{
Лазер асистирана увулопалатопластика
}

\author{
(Laser-Assisted Uvulopalatoplasty - LAUP)
}

\author{
Св. Василева', Пл. Недев², С. Стоянов', М. Милков², Р. Бенчев \\ 'Медицински институт на МВР, София

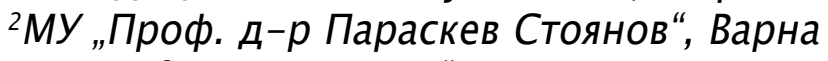 \\ ${ }_{3}^{3}$ „ХЛ КЛИНИК“АД, София
}

\section{Резюме:}

Хирургичните възможности за лечение на пациенти, страдащи от разстройства на дишането по време на сън, бележат значително развитие през последните десетилетия. Към днешна дата съществува огромно разнообразие от методи и техники, разработени за справяне с този проблем. С навлизането в ежедневната практика на лазерната и радиочестотната хирургия стана възможна т.нар. „минимално инвазивна хирургия “, която може да се извършва и под местна анестезия, в амбулаторни условия.

Целта на настоящата презентация е да представим един от най-съвременните методи - Laser-Assisted Uvulopalatoplasty (LAUP). Това е сравнително нов метод и опитът с него в България не е голям. За много от специалистите в страната този метод е в значителна степен непознат както като техническо изпълнение, така и като възможности. Целта на настоящата публикация е да представим този полезен, относително лесен за изпълнение и с висока ефективност метод и да способстваме повече български оториноларинголози да започнат да го използват в ежедневната си практика.

\section{Въведение}

Лазер асистираната увулопалатопластика (LAUP) е представена за първи път от Dr Yves-Victor Kamami през 1986 г., но широко приложение в практиката намира от 1993 год. През 1997 г. American Academy of Otolaryngology - Head and Neck Surgery включва LAUP към процедурите за лечение на OSA ${ }^{a}$.

Този тип оперативна намеса, като самостоятелна процедура, е индицирана при пациенти с т. нар. „Плосък тип велофаринкс“, т.е. когато обструкцията на горните дихателни пътища изхожда приоритетно от мекото небце - увулата и/или дъгите (т.е. липсва обструкция от страна на езика - FTP I - II). При неблагоприятна позиция на езика (FTP III - IV) LAUP може да бъде елемент от хирургия на няколко нива - съчетава се с процедури за намаляване на обструкцията на ниво хипофаринкс. По литературни данни този тип хирургия е неефективна при хипертрофия на сливиците или обструкция от латералните колони

\section{Хирургична техника}

От литературата познаваме два основни метода за изпълнение на този тип оперативна намеса. Първият, известен като Френски метод (представен от Kamami през 1990 год. ${ }^{c}$ ), е подходящ при пациенти, при които това е първа намеса върху мекото небце. 


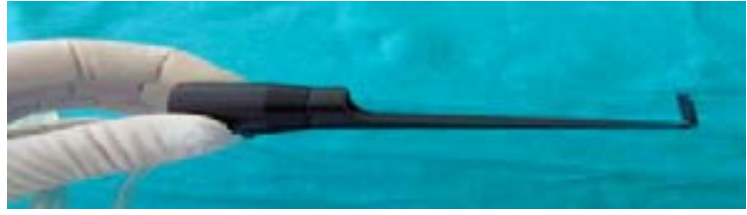

Фиг. 1. Накрайник за меко небце и увула (c „backstop” за протекция на лигавицата по задната стена на мезофаринкса)

Ние работим с $\mathrm{CO}_{2}$ LASER на фирма DEKA (Фиг. 1 и 2) най-често по метода на Kamami.

Етапи на осъществяване на оперативната намеca:

1. Местна анестезия: Операцията може да бъде извършвана и под местна анестезия. 5-10 минути преди намесата се впръсква локално лидокаин спрей (двукратно).

Непосредствено преди вапоризацията, дори при работа под обща анестезия, с цел да бъде намалено кървенето в оперативното поле се прави проводна анестезия с адреналин и лидокаин $(1: 100000)$ в 6 пункта - 2 над увулата по срединната линия на мекото небце и в по 2 пункта латерално над предните небни дъги.

2. Започва се работа с настройвания от апаратурата режим - 15W (фиг. 3), постоянна вълна. При по-масивни небца мощността на лазера може да бъде увеличена ръчно до 20-25W.

3. Евапорация на купуловидни фрагменти от лигавицата на задните небни дъги до нивото на мускулите и на до 1 см странично от мъжеца. Част от тонзиларните дъги и сли-

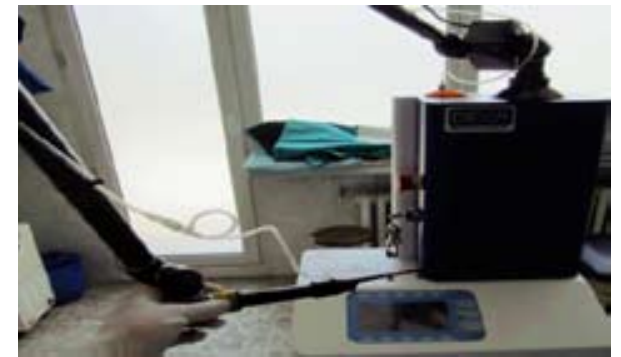

Фиг. 2. $\mathrm{CO}_{2}$ LASER „DEKA” с прикачена представка за меко небце

виците също могат да бъдат вапоризирани (фиг. 4).

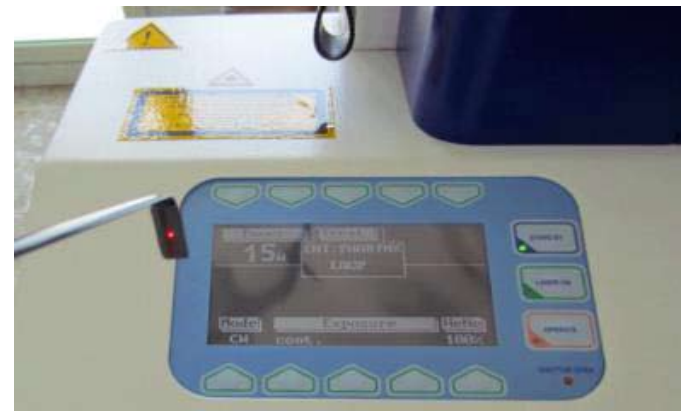

Фиг. 3. Монитор на $\mathrm{CO}_{2}$ LASER. Параметри за LAÜP.
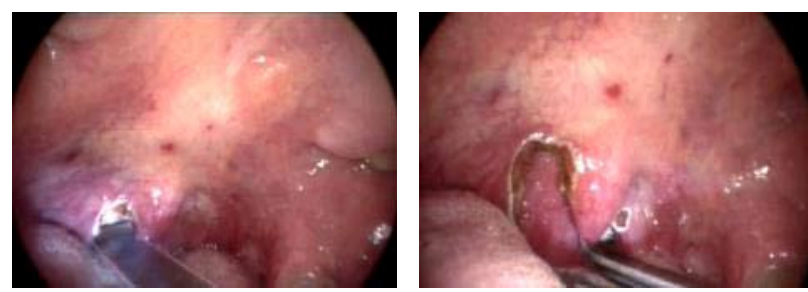

Фиг. 4. Евапорация на задните небни дъги

4. Резецират се и дисталните $2 / 3$ на увулата - поетапно от двете страни (Фиг. 5).
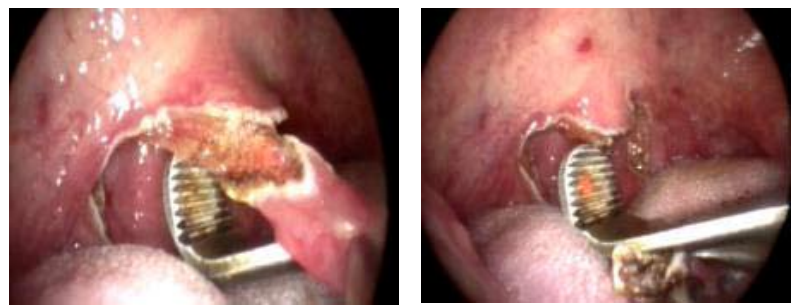

Фиг. 5. Резекция на увулата
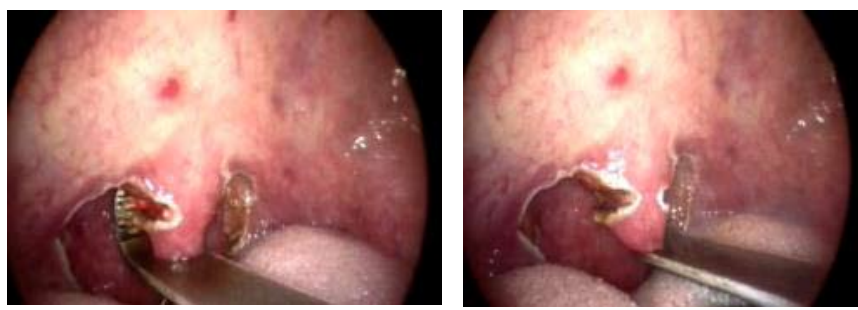

Фиг. 6. Британски метод - (Фигурата е от Friedman M.: Sleep apnea and snoring: surgical and non-surgical therapy 2009

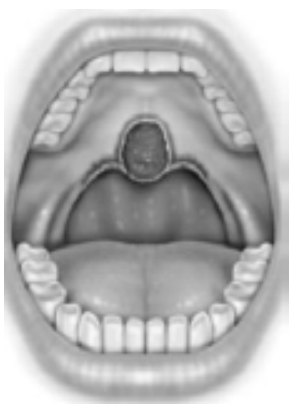


При този тип оперативни намеси кървенето обикновено е слабо и се овладява лесно с разсеян лазерен лъч. Изключително рядко има кървене, което да налага използване на допълнителни методи за хемостаза. Заздравяването на раневата повърхност става между втората и третата седмица (14 и 21 дни), а пациентите имат болка (макар и поносима) до седмица след оперативната намеса. При необходимост процедурата може да се повтаря.

През 1994 год. Woolford и Farrington ' представят модификаиия, известна като Британска техника (Фиг. 6). Най-често тя намира приложение като втори етап, след осъществена LAUP по метода на Kamami.

\section{Хирургична техника}

Използва се лазерен лъч с мощност 20W и обработваната повърхност е около 2 см. Евапорира се лента от лигавицата на увулата и мекото небце до нивото на твърдото небце. Подлежащите мускули на мекото небце и бялата линия не бива да бъдат засягани. Повърхността се оставя да контрахира и да образува цикатрикс вторично, което води до допълнително втвърдяване на небцето.

Предимство на LAUP е, че при евапорация с лазер освен отстраняването на част от тьканта се предизвиква и особена форма на повърхностно възпаление в областта на мекото небие и фаринкса, което впоследствие води до формиране на иентростремителна фиброза c упльтняване на тъканите, резултиращо в допълнително намаляване на способността им да вибрират. Важно е да се избягва формиране на циркумферентни раневи повърхности и обгаряне на широка площ извън полето, защото това може да доведе до контрактури във фаринкса и последваща стеноза.

LAUP може да се извършва и под местна анестезия и в амбулаторни условия ${ }^{\mathrm{e}, \mathrm{f}}$. Проблем в този случай е фактьт, че в ранния следоперативен период е възможно да се появи оток в горните дихателни пьтища ${ }^{\mathrm{g}}$, което налага постоперативно наблюдение и мониторинг на кислородната сатурация за период от поне 48 часа. В тази връзка все повече автори препоръчват LAUP да се извършва в условията на стационарно лечение. Процедурата е с по-слаба болезненост в сравнение с класическата увулофарингопа- латопластика (UPPP), но през първите няколко дни при повечето пациенти се налага да бъдат използвани обезболяващи. При съпътстващ гастро-езофагеален рефлукс е задължително да се включат и антиациди. Пациентите е желателно да бъдат проследявани и преоценявани поне до 6 седмици след операцията. По литературни данни за оптимален ефект от LAUP често са необходими между 2 и 4 процедури през 4-6 седмици. Към този момент не ни се е налагало да повтаряме процедурата.

\section{Усложнения}

LAUP е техника с висока степен на безопасност. Най-често докладваните усложнения са: усещане за чуждо тяло, сухота във фаринкca, постоперативно кървене, инфекции, палатинална или велофарингеална недостатъчност и много рядко - назо-фарингеална стеноза. Средно процентът на усложнения е около $3,5 \%$ h. Най-чести са хеморагиите - 2-3\%, следвани от инфекции - $1 \%$, велофарингеална недостатъчност е наблюдавана при по-малко от $1 \%$ от пациентите, като по-често е преходна. Описани са и случаи на велофарингеална стеноза, но честотата е много ниска.

\section{Ефективност}

През 1990 год. Кататі докладва 77\% пълно или почти пълно изчезване на хъркането при пациенти без апноични паузи по време на сън ${ }^{\mathrm{i}}$. През 1995 год. Walker at all. докладват 60\% изчезване на хъркането и $29 \%$ сигнификантно намаление при обследване на 170 пациенти ${ }^{\mathrm{i}}$. Средно докладваният процент на успеваемост на процедурата по отношение на хабитуалното хъркане е между 60 и 84\%. Редица автори докладват подобен процент на сигнификантно повишаване на качеството на живот на пациентите след LAUP' шенията с партньора ( $72 \%$ удовлетворение на партньора) ${ }^{\mathrm{k}}$.

За добри резултати при лечение на OSA от много важно значение е подборьт на пациентите. При правилно поставени индикации докладваната ефективност, сьгласно критериите на Шер, е $57 \%$ за лека степен на OSA, 48\% при средна и 46\% при пациенти с тежка по степен OSA ${ }^{1, m}$.

Съществуват и доклади за значително по-нисък процент на успеваемост (обективна, след поли- 
сомнография) $-27 \%$ n , като се подчертава, че субективната позитивна оценка на пациентите значително надхвърля обективните резултати.

Налични са и доклади за обективно влошавате на състоянието на пациенти с OSA след LAUP - Finkelstein and colleagues през 2002 год. при обследване на 26 пациенти докладват 31\% повишаване на RDI постоперативно. Те изказват предположението, че причината е велофарингеална стеноза като следствие от прогресивна палатинална фиброза ${ }^{\circ}$.

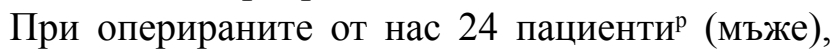
между 6 и 9 месец след операциите, отчетохме:

- 92\% ефективност по отношение на хъркането (субективна оценка от анамнеза и въпросници). Само при двама пациенти партньорите съобщиха, че макар и с по-ниськ интензитет, хъркане персистира през повече от 3-4 нощи през седмицата (при хъркане всяка вечер преди операцията).

- 6 от пациентите преди операциите докладваха повишена сънливост през деня (ESS над 10). След намесата ESS при всички беше под 10 - т.е. $100 \%$ ефективност на база субективна оценка по отношение на сьнливостта през деня.

- Разгледаните 6 пациенти преди операциите проведоха и полисомнография (полиграфия) и при всички бе отчетен АНІ над 5 (от 9 до 28). След операциите редукция на АНІ с 50 и повече процента постигнахме при 4 от тези пациенти (67\%).

При оперираните от нас пациенти не сме имали случаи на постоперативно кървене. Болката беше по-изразена, отколкото след радиочестотна увулопалатопластика (RF UPP), но значително по-слаба от тази след UPPP. Нужда от обезбо- ляващи пациентите имаха през първите 1-2 дни. Седем пациенти до 4-6 ден след операциите имаха по-изразен оток на мекото небце и съобщиха за усещане за чуждо тяло, което отшумя спонтанно при 4 от тях, а при трима поставяхме кортикостероид по схема за период от 3 дни. При двама пациенти установихме инфектиране на раневата повърхност съответно на 5. и на 7. ден след оперативната намеса (в домашни условия), но възпалението беше лесно овладяно с локални антисептични разтвори и перорален антибиотик.

\section{Заключение}

Нашият опит с LAUP все още не е достатъчно голям, за да бъдем категорични и убедителни в заключенията си. Въпреки това, на база резултатите от това проучване можем да излезем със заключенията, че: LAUP е относително лесна за изпълнение техника, с висока степен на безопасност, нисък риск от постоперативни усложнения, лесно поносима от пациентите и с висок процент на ефективност при правилно поставени индикации.

Изключително важно при този тип хирургия е да се избягват ициккумерентните ичикатрикси. Дългосрочната удовлетвореност след LAUP е в правопропорционална зависимост от 2 фактори - правилен подбор на пациенти и прецизно изпьлнение.

LAUP е техника, доказала ефективността си при лечение на хъркане и сънна апнея. Фактори, лимитиращи използването ѝ в клиничната практика, са намаляването на ефекта с времето и евентуалната необходимост процедурата да се повтаря.

\section{Литература:}

a Walker RP. Laser assisted uvulopalatoplasty. In: Snoring and Obstructive Sleep Apnea, $3^{\text {rd }}$ edn. 2003: 144-50

b Finkelstein Y, Shapiro-Feinberg M, Stein G et al: Uvulopalatopharyngoplasty vs laser-assisted uvulopalatoplasty. Arch Otolaryngol Head Neck Surg 123: 265-276, 1997

c Kamami Y . Laser CO 2 for snoring, preliminary results . Acta Otorhinolaryngol Belg 1990; 44: 45-56

d Woolford T, Farrington T. Laser-assisted uvulopalatoplasty - the British method. Oper Tech Otolaryngol Head Neck Surg 1994; 5: 292-93

e Remacle M, Betsch C, Lawson G et al: A new technique for laser-assisted uvulo(palato)plasty decision-tree analysis and results. Laryngoscope 109:763-768, 1999

f Mickelson SA, Ahuja A: Short-term objective and long-term subjective results of laser

g Esclamado RM, Glenn MG, McCulloch TM, et al. Perioperative complications and risk factors in the surgical treatment of obstructive sleep apnea syndrome. Laryngoscope 1989; 99: 1125-29

h Walker RP, Gopalsami C . Laser-assisted uvulopalatoplasty: postoperative complications . Laryngoscope 1996; $106: 834-8$

i Walker RP, Grigg-Damberger MM, Gopalsami C, et al. Laserassisted uvuloplasty for snoring and obstructive sleep apnea: resultsin 170 patients. Laryngoscope 1995; $105: 938-43$ 
j Armstrong MW, Wallace CL, Marais J. The effect of surgery upon the quality of life in snoring patients and their partners: a between-subjects case-controlled trial . Clin Otolaryngol Allied Sci 1999; 24: 510-22

k Prasad KR, Premraj K, Kent SE, Reddy KT. Surgery for snoring:are partners satisfied in the long run? Clin Otolaryngol Allied Sci 2003; 28: 497-502

1 Mickelson SA, Ahuja A. Short-term objective and long-term subjective results of laser-assisted uvuloplatoplasty for obstructive sleep apnea. Laryngoscope 1999; 109: 362-7

m Walker RP, Garrity T, Gopalsami C. Early polysomnographic findings and long-term subjective results in sleep apnea patients treated with laser-assisted uvulopalatoplasty. Laryngoscope 1999; 109: 1438-41

n Ryan CF, Love LL. Unpredictable results of laser assisted uvulopalatoplasty in the treatment of obstructive sleep apnoea. Thorax 2000; 55: 399-404

o Finkelstein Y, Stein G, Ophir D, et al. Laser-assisted uvulopalatoplasty for the management of obstructive sleep apnea Arch Otolaryngol Head Neck Surg 2002; 128: 429-34

p Василева Св.: Съвременни методи за оперативно лечение на разстройствата на дишането по време на сън - Дисертационен труд 2014; 22-24: $87-88$ 